\title{
Prevalence of the Complications of Diabetes in Developing Countries
}

\author{
Sarpong C, Nsiah K, Mensah F. O, Agyeman-Duah E*, Ahmed F. D \\ Department of Biochemistry and Biotechnology, College of Science, Kwame Nkrumah University of Science and \\ Technology, Kumasi-Ghana. \\ *Corresponding Author: Agyeman-Duah E, Department of Biochemistry and Biotechnology, College of \\ Science, Kwame Nkrumah University of Science and Technology, Kumasi-Ghana, E-mail: saxcess21@gmail.com
}

Received: 10 May 2017; Accepted: 18 July 2017; Published: 17 August 2017

\begin{abstract}
Diabetes mellitus is a medical condition which arises when there is an uncontrollably high level of glucose in the blood. It is a carbohydrate metabolism disorder which also affects protein and fatty acid metabolisms. Several complications result from diabetes as a result of poor glycemic control. This project aimed at determining the various complications developed by diabetics who visited the Diabetics Clinic of the Komfo Anokye Teaching Hospital (KATH), Kumasi, Ghana, from January to December, 2013. It involved 475 diabetes with an age range of 11 to 100 years with modal age range being greater than 60 years $(56.8 \%)$ and a mean age of $63.8 \pm 3.2$ years. It comprised of $45.7 \%$ males and $54.3 \%$ females.
\end{abstract}

The predominant diabetes type was type 2 (affecting 269 patients) followed by unclassified diabetes type (affecting 188 patients) and then type 1 diabetes (also affecting 18 patients). Complications were found in 365 (76.8\%) patients with most of these complications occurring in females who were above 40 years. The most prevalent complication was hypoglycemia, which affected 180 patients (37.9\%). Peripheral circulatory complication affected 18 patients (3.8\%), renal complication affected 123 patients (25.9\%) and ketoacidosis also affected 42 patients (8.8\%) whilst two patients (0.4\%) suffered multiple complications. These complications caused 57 deaths (12\%) and these deaths were most prevalent in unclassified diabetes.

Keywords: Diabetes Mellitus; Complication; Circulatory; Renal; Ketoacidosis 


\section{Introduction}

Diabetes affects 246 million people in the world and the expectation is that by 2025, about 380 million people will be diabetics and its prevalence will take place in developing countries [1]. The global estimate of the number of diabetics in Africa was approximately 12 million people (adults of age group 20-79 years) in 2010 and the expectation is that by 2030, about 23 million Africans will be diabetes [2].

Several efforts have been initiated in creating the awareness of diabetes in Ghana. These include setting up National Diabetes Association, organizing celebration of World Diabetes Days and organizing Diabetes Conferences. For instance, the Ministry of Health and the Ghana National Diabetes Association under the auspices of Otumfour Osei Tutu II, the Asantehene, organized celebration of World Diabetes Day which took place at the Golden Tulip Hotel in Kumasi in November, 2013 [3]. The prevalence of the disease, however, is still on the increase, with Ghana recording about 4 million diabetics as of 2012 which represent 17.00\% of the total population [4].

Diabetes and its associated complications have become a public health problem of considerable magnitude. The effects of diabetes can be devastating if blood sugars are not controlled. In Sub-Saharan Africa, 10.0-15.0\% of diabetic patients develop foot ulcers at some stage of their lives and nearly $50.00 \%$ of all diabetes-related admissions are due to diabetic foot problems [5]. The prevalence of diabetic foot ulcer ranged between $1.0 \%$ and $4.10 \%$ in the United States (US), $20.40 \%$ in the Netherlands and $20.00 \%$ in Iran among diabetics. The prevalence was also about $4.60 \%$ among diabetics in Kenya, and hospital-based studies demonstrated that the prevalence of limb ulceration was between $11.70 \%$ and $19.10 \%$ among individuals with diabetes in Nigeria [6].

In Ghana, diabetic patients with high levels of glycosylated hemoglobin are prone to micro-vascular complications like nephropathy and retinopathy and patients with poor glycemic control are usually the sufferers of these complications [7]. The complications posed by diabetes have substantial reduction in quality of life and it is also associated with significant morbidity and high mortality rates [8]. The present study is to determine the prevalence of complications in diabetes mellitus among diabetics who visit the Diabetics Clinic of the Komfo Anokye Teaching Hospital (KATH).

\section{Methodology}

The study area was the Diabetics Clinic of the Komfo Anokye Teaching Hospital, Kumasi. This is a retrospective review of 475 cases of patients suffering from different types of diabetes and also developed different complications. Data was collected from disease classification cards obtained from the Main Records Unit of the hospital. Relevant data for the present study were codes, ages, sex and complications of diabetics who had visited the hospital from January to December, 2013.

Data acquired was entered and then analyzed using Microsoft Excel 2010 and SPSS version 16.0. Two way ANOVA from SPSS was used for the analysis with a confidence level of 95\%. Ethical approval was obtained from 
the appropriate Units before commencement of present study.

\section{Results}

Data for a total of 475 diabetics were obtained of whom 217 (45.7\%) were males and 258 (54.3\%) were females. Different types of diabetes existed among varying numbers of males and females. Table 1 shows the number of male and female diabetics who visited the hospital from January to December, 2013.

Table 1 further illustrates the ages of the diabetics recorded, where 2.5\% were under age 20, $13.9 \%$ were between 19 and 39 years, 26.7\% were between 40 and 59 years and 56.8\% were also above 60 years.

\begin{tabular}{|c|c|c|c|c|}
\hline & $\begin{array}{l}\text { Type } 1 \\
(n=18)\end{array}$ & $\begin{array}{c}\text { Type } 2 \\
(n=269)\end{array}$ & $\begin{array}{c}\text { Unclassified } \\
(n=188)\end{array}$ & $\begin{array}{c}\text { Total } \\
(n=475)\end{array}$ \\
\hline $\begin{array}{|cc|}\text { SEX } & \\
& \text { Males } \\
& \text { Females } \\
\end{array}$ & $\begin{array}{l}09(50.0 \%) \\
09(50.0 \%)\end{array}$ & $\begin{array}{l}133(49.4 \%) \\
136(50.6 \%)\end{array}$ & $\begin{array}{c}\text { 75(39.9\%) } \\
113(60.1 \%)\end{array}$ & $\begin{array}{l}217(45.7 \%) \\
258(54.3 \%)\end{array}$ \\
\hline $\begin{array}{|cc|}\text { AGE } & \\
& <20 \\
& 20-40 \\
& 41-60 \\
& >60 \\
& \text { Total } \\
\end{array}$ & $\begin{array}{c}05(27.8 \%) \\
07(38.9 \%) \\
04(22.2 \%) \\
02(11.1 \%) \\
18\end{array}$ & $\begin{array}{c}03(1.1 \%) \\
27(10.0 \%) \\
61(22.7 \%) \\
178(66.2 \%) \\
269\end{array}$ & $\begin{array}{c}04(2.1 \%) \\
32(17.0 \%) \\
62(33.0 \%) \\
90(47.9 \%) \\
188\end{array}$ & $\begin{array}{c}12(2.5 \%) \\
66(13.9 \%) \\
127(26.7 \%) \\
270(56.8 \%) \\
475\end{array}$ \\
\hline
\end{tabular}

Table 1: Age and sex distribution among the various types of diabetes

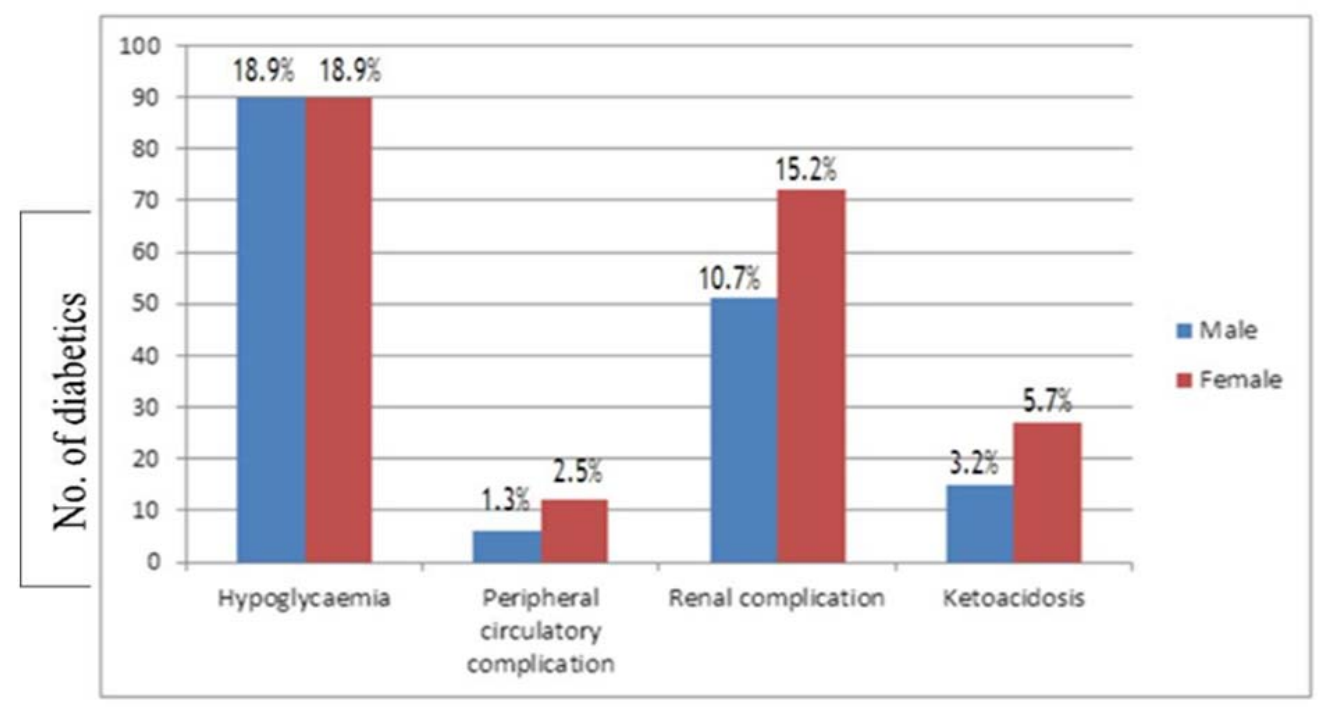

Figure 1: Complications and their prevalence amongst diabetes patients

Figure 1 represents the various complications recorded among the diabetics. It is also a representation which compares the complications in males to females, where $34.1 \%$ of males developed complications, compared to 
42.7\% of females who also developed complications. Hypoglycemia affected equal numbers of males and females, whilst the other complications affected a greater number of females than males.

Two patients (0.4\%) out of the total number of diabetics developed multiple complications and 110 patients (23.2\%) out of the total number of diabetics did not develop any form of complication.

The prevalence of complications resulting from diabetes among the different diabetes types is illustrated in Table 2 . This represents the types of diabetes and their complications prevalence. The most prevalent complication existed among type 1 diabetes where all of them developed some form of complication. The percentage of unclassified diabetics with complications was not significantly $(\mathrm{p}=0.13)$ higher than those without complications. The percentage of type 2 diabetics with complications was however significantly ( $\mathrm{p}=0.04$ ) higher than those without complications. The prevalence of the various complications among the diabetes types varied. Type 1 and type 2 diabetics had hypoglycemia as the most prevalent complications, whereas there were no cases of renal complication and ketoacidosis in type 1 diabetes. Unclassified diabetes, however, had renal complication as the most prevalent complication.

\begin{tabular}{|c|c|c|c|c|c|c|}
\hline \multirow{2}{*}{ Gender } & \multicolumn{2}{|c|}{ TYPE 1 (n=18) } & \multicolumn{2}{c|}{ TYPE 2 (n=269) } & \multicolumn{2}{c|}{$\begin{array}{c}\text { UNCLASSIFIED } \\
(\mathbf{n}=188)\end{array}$} \\
\cline { 2 - 7 } & C & NC & C & NC & C & NC \\
\hline Male & $9(50.0 \%)$ & - & $79(29.4 \%)$ & $54(20.1 \%)$ & $74(39.4 \%)$ & $1(0.5 \%)$ \\
\hline Female & $9(50.0 \%)$ & - & $82(30.4 \%)$ & $54(20.1 \%)$ & $\begin{array}{c}112 \\
(59.6 \%)\end{array}$ & $1(0.5 \%)$ \\
\hline
\end{tabular}

Table 2: Diabetes type and prevalence of their complications

$\mathrm{C}=$ Complication; $\mathrm{NC}=$ No complication

\section{Discussion}

The number of diabetic females was higher than male diabetics and this correlates with the studies by Darkwa [9] in the Cape Coast Metropolis. This can be attributed to fact that females practice less diabetes management methods such as regular body exercise and intake of low calorie diets. Majority of diabetes patients were above 60 years (56.8\%) as a result of the fact that as people age, functions of their organs decline because of decreased cellular metabolic activities [10]. The higher prevalence of diabetes in females may have resulted from more females visiting the hospital in 2013, compared to the number of males who also visited the hospital. The greater number of type 2 diabetics in the study is in accordance with the 1999 WHO report [11]. The most prevalent diabetes type being type 2 diabetes mellitus (non-insulin dependent diabetes mellitus) typically associated with diet, obesity and physical inactivity [11] occurred amongst 269 diabetes patients (56.6\%).This greater prevalence of type 2 diabetes can be attributed to adoption of western lifestyle such as diet. 
Aside the two well-known type 1 and type 2 diabetes, an unclassified type of diabetes was also found which affected $39.6 \%$ of the total number of diabetics. In Africa, presence of atypical forms of diabetes makes it difficult to categorize patients as having either type 1 or type 2 diabetes based on usual clinical criteria [12]. For example, a study by Abdukadir et al [13] in Ethiopia reported that besides the two main types of diabetes is tropical pancreatic diabetes, characterized by early-onset, non-ketotic diabetes in underweight patients with high insulin requirement. Several complications develop in diabetes. The extent of the complications correlates positively with the duration of the diabetes and the effectiveness of management methods aimed at maintaining blood glucose levels within the normal range. These complications may be acute or chronic depending on the duration of the diabetes.

Approximately $76.4 \%$ of the diabetics had some form of complication. These complications included hypoglycemia, which ultimately led to coma. Peripheral circulatory complications, renal complications and ketoacidosis were also prevalent. Some people, however suffered from multiple complications accounting for $0.4 \%$ of the overall patients. These multiple complications were as a result of very high levels of glucose in the blood. Some of the patients also did not develop any form of complication (23.2\%) which can be attributed to either the short duration of the diabetes or there was effective glycemic control, averting development of complications.

The most prevalent complication in this study was hypoglycemia which occurred (an acute complication) amongst 180 diabetics (37.9\%). This complication was prevalent in type 1 diabetics which can be attributed to the fact that insulin injection being the main method of its management caused an abnormally high absorption of glucose from the blood. This therefore caused the blood glucose levels falling below normal, causing hypoglycemia. This hypoglycemia occurred in same numbers of males compared to females, although the expectation was a high prevalence in males because of high metabolic rates in males. The complication was also prevalent in patients of age group 20-40 years because of the higher physical activity levels in these people. Hypoglycemia also caused deaths amongst 20 diabeties.

The least prevalent complication was peripheral circulatory complication, that is, atherosclerosis of the arteries of the limbs especially the legs. This condition occurred amongst 18 patients 35 (3.8\%) causing only a single death. This least prevalence of peripheral circulatory complication might be due to the increased education to diabetics on foot care.

Other complications such as renal complication and ketoacidosis were also prevalent. These complications however did not occur amongst type 1 diabetics. Renal complication with microalbuminuria being an early marker existed amongst 123 patients (25.9\%) and this was prevalent in the age group greater than 60 years. Ketoacidosis caused by elevated levels of ketone bodies in the blood is as a result of fatty acid metabolism. When glucose is not absorbed from the blood into the cells as a result of insulin ineffectiveness the body starts to break down fatty acids to generate energy. The continuous breakdown of fatty acids causes elevated levels of ketone bodies in the blood 
ultimately leading to ketoacidosis. This condition existed amongst 42 patients (8.8\%) and was more prevalent in unclassified diabetes.

\section{Conclusion}

In the study, $76.8 \%$ of the diabetics had some types of complication including hypoglycemia (37.9\%), peripheral circulatory complication (3.8\%), renal complication (25.9\%), ketoacidosis (8.8\%) and multiple complications (0.4\%). These complications were prevalent in females (42.7\%) compared to the males (34.1\%). These complications also caused 57 deaths (12\%) and these deaths were prevalent in unclassified diabetics.

\section{Acknowledgement}

The authors would like to acknowledge the staff of the Diabetics Clinic and Main Records Unit of the Komfo Anokye Teaching Hospital, Kumasi for their assistance in this project.

\section{Conflict of Interest}

No conflict of interest declared by the authors.

\section{References}

1 Ziaei-Rad M, Vahdaninia M, Montazeri A. Sexual dysfunctions in patients with diabetes: a study from Iran. Reproductive Biology and Endocrinology 50 (2010): 1-8.

2 http://www.idf.org/diabetes 2nd October, 2013.

3 http://www.ghana.gov.gh 11th February, 2014.

4 Jones S. Prevention of Hypertension and Diabetes in Ghana. Showcase of Undergraduate Research Excellence. 31 (2013): 1-4.

5 Agwu E, Dafiewhare EO, Ekanem PE. Possible diabetic-foot complications in Sub-Saharan Africa. Global Perspective on Diabetic Foot Ulcerations. (2011): 1-14.

6 Desalu OO, et al. Diabetic foot care: Self-Reported Knowledge and Practice among Patients Attending Three Tertiary Hospital in Nigeria. Ghana Medical Journal. 45 (2011): 60-65.

7 Arthur FKN, et al. Fasting Blood Glucose and Glycosylated Haemoglobin Levels in Randomly Selected Ghanaian Diabetic Patients-The Clinical Implications. Journal of Science and Technology 25 (2006): 7-11.

8 http://ezinearticles.com/?Diabetes. What Are the Effects?\&id=1454273 29th September, 2013.

9 Darkwa S. Prevalence of diabetes mellitus and resources available for its management in the Cape Coast Metropolis. ISABB Journal of Health and Environmental Sciences 1 (2011): 1-7.

10 Wild SH, et al. Global Prevalence of Diabetes: Estimates for the Year 2000 and Projections for 2030 Response to Rathman and Gaini. Diabetes Care. 27 (2004): 2569-25

11 WHO. Definition, Diagnosis and Classification of Diabetes Mellitus and its Complications. Part 1: Diagnosis and Classification of Diabetes mellitus (1999): 1-49. 
12 Sleire L. Diabetes: A Neglected Disease in Sub-Saharan Africa. A comparative study between Rwanda and Norway (2011): 1-65.

13 Perret J, Nguemby-Mbina C. Characteristics of the development of insulin need in primary diabetes of adults in Gabon. Ann SocBelg Med Trop 77 (1991):243-249.

14 Abdukadir J, et al., The clinical and hormonal (C-peptide and glucagons) profile and liability to ketoacidosis during nutritional rehabilitation in Ethopian patients with malnutrition-related diabetes mellitus. Diabetologia. 33 (1990): 222-237. 